\title{
Adjuvant Chemotherapy Following Combined Induction Chemotherapy and Concurrent Chemoradiotherapy Improves Survival in N2-3-positive Nasopharyngeal Carcinoma Patients
}

\section{Hao-Yun Tao}

Guangzhou Medical University Affiliated Cancer Hospital

Hui Liu

Guangzhou Medical University Affiliated Cancer Hospital

\section{Cai-Xian He}

Guangzhou Medical University Affiliated Cancer Hospital

\section{Ran Li}

Shenzhen Longhua New District People's Hospital

\section{Kun-Peng Du}

Guangzhou Medical University Affiliated Cancer Hospital

Ya-Wei Yuan

Guangzhou Medical University Affiliated Cancer Hospital

Rong-Hui Zheng ( $\nabla$ zhengronghui@gzhmu.edu.cn )

Guangzhou Medical University Affiliated Cancer Hospital

\section{Research Article}

Keywords: Concurrent chemoradiotherapy, nasopharyngeal carcinoma, propensity score matching, adjuvant chemotherapy, induction chemotherapy

Posted Date: September 7th, 2021

DOI: https://doi.org/10.21203/rs.3.rs-848732/v1

License: (c) (i) This work is licensed under a Creative Commons Attribution 4.0 International License. Read Full License

Version of Record: A version of this preprint was published at Journal of Cancer Research and Clinical Oncology on November 25th, 2021. See the published version at https://doi.org/10.1007/s00432-021-03846-6. 


\section{Abstract}

Objective: This study aimed to explore the clinical value of adjuvant chemotherapy (ACT) in locoregionally advanced nasopharyngeal carcinoma (LANC) following concurrent chemoradiotherapy (CCRT) and induction chemotherapy (ICT).

Methods: We included 839 newly diagnosed LANC patients in the study. ICT plus CCRT (ICT+CCRT group) was administered to 443 patients and 396 patients who received ACT after receiving ICT plus CCRT (ICT+CCRT+ACT group). Univariate and multivariate Cox regression analyses were carried out in this study. Furthermore, to balance the study and control groups, propensity score matching (PSM) was applied.

Results: 373 pairs of LANC patients were obtained after the PSM analysis. We found that ACT following ICT+CCRT had no significant effect on improving the survival of LANC patients. By further exploring the ICT+CCRT+ACT regimen, we excluded N0-1-positive patients and performed PSM in the ICT+CCRT and ICT+CCRT+ACT groups again. Each group consisted of 237 patients. Kaplan-Meier analysis revealed that there was a difference between the ICT+CCRT and ICT+CCRT+ACT groups in terms of the 5-year overall survival (OS) $(78.9 \%$ vs. 85.0\%, P = 0.034), disease-free survival (DFS) $(73.4 \%$ vs. $81.7 \%, P=0.029)$, and distant metastasis-free survival (DMFS) $(84.9 \%$ vs. $76.0 \%, P=0.019)$. In addition, the ICT+CCRT+ACT group had a higher incidence of grade 3-4 acute leukocytopenia/neutropenia.

Conclusion: Compared with ICT+CCRT, ACT following ICT plus CCRT can reduce distant metastasis of N2-3-positive LANC and improve the OS and DFS of these patients, thus demonstrating higher clinical feasibility.

\section{Introduction}

Nasopharyngeal carcinoma (NPC) is endemic in southern Asia, and distant metastasis is the leading cause of mortality[1]. NPC clinical symptoms and signs are often nonspecific, so more than $60 \%$ of patients have locally advanced disease when diagnosed [2]. Because of its complicated anatomical position and strong radiosensitivity, radiotherapy remains the main treatment for NPC. However, for locally advanced NPC patients (LANC), radiotherapy alone may have a limited effect [3].

The result of the intergroup 0099 study was to demonstrate the clinical value of chemotherapy for patients with LANC [4]. Concurrent chemoradiotherapy (CCRT) increases survival and lowers the incidence of distant metastasis in LANC patients, according to several prospective studies conducted in NPC epidemic regions [5, 6]. Subsequently, Sun et al. [7] found that compared to CCRT alone, combining induction chemotherapy (ICT) with CCRT improved 3year failure-free survival (FFS) in LANC patients. Thus, the National Comprehensive Cancer Network Guidelines (NCCN) recommend CCRT following ICT as one of the best therapeutic regimens for LANC patients (category 2A). [7]. ICT followed by definitive CCRT could improve clinical outcomes, but approximately $30 \%$ of patients will subsequently develop recurrence and/or distant metastasis [8-12].

Several studies have shown that adjuvant chemotherapy (ACT) following neoadjuvant therapy and surgery can markedly improve local control and OS in non-small-cell lung carcinomas [13-15]. In an individual patient data network meta-analysis of 5144 patients, ACT following CCRT achieved the best survival benefit [16]. Zou et al. [17] demonstrated the safety of ACT following ICT plus CCRT (ICT + CCRT + ACT) in LANC patients; however, this report lacked conclusions regarding a comparison with the ICT + CCRT regimen. Therefore, the objective of this study was to further explore the efficacy of ICT plus CCRT followed by ACT in LANC patients. 


\section{Patient Selection And Methods \\ Eligibility criteria}

Patients were restaged using the AJCC/UICC 8th edition. The following were the criteria for inclusion: (1) WHO type I, II, or III pathologic NPC diagnosis; (2) completed definitive intensity-modulated radiotherapy (IMRT); (3) Karnofsky scale score $\geq 70$; (4) clinical stage III/IVa; (5) administered a regimen of cisplatin/nedaplatin plus docetaxel (TP) induction chemotherapy (ICT) followed by concurrent chemoradiotherapy (CCRT) with or without TP adjuvant chemotherapy (ACT). The criteria for exclusion included (1) a history of prior cancers and (2) signs of severe heart, lung, liver, renal, and other critical organ malfunction. Between January 2011 and December 2016, 839 patients were screened and admitted to the Department of Radiation Oncology at Guangzhou Medical University's Affiliated Cancer Hospital \& Institute. In this study, we divided the subjects into two groups: the ICT + CCRT group ( $n=443$ ) received TP induction chemotherapy followed by CCRT, and the ICT + CCRT + ACT group $(n=396)$ received TP adjuvant chemotherapy after TP induction chemotherapy with CCRT. Our hospital's Medical Ethics Committee authorized this retrospective investigation.

The details of this study are shown in Fig. 1.

\section{Induction chemotherapy}

TP induction chemotherapy was administered to all patients. Cisplatin/nedaplatin $\left(75-80 \mathrm{mg} / \mathrm{m}^{2}\right.$, day 1$)$ and docetaxel $\left(75 \mathrm{mg} / \mathrm{m}^{2}\right.$, day 1$)$ were used in the TP regimen. Before CCRT, the regimen was repeated every three weeks.

\section{Concurrent chemoradiotherapy}

All patients underwent radical IMRT. We established target volumes based on the ICRU 62 study. We defined the main cervical lymph node tumor volume (GTVnd) and gross tumor volume (GTVnx) of the whole macroscopic tumor as determined by magnetic resonance imaging (MRI), physical exams, and computed tomography (CT). The recommended radiation doses were 70-74 Gy, 66-68 Gy, 60-66 Gy, and 54-56 Gy for the GTVnx, GTVnd, high-risk clinical target volume (CTV1) and low-risk clinical target volume (CTV2), respectively. In total, 31 to 33 fractions were given to each patient. Cisplatin/nedaplatin $\left(75-80 \mathrm{mg} / \mathrm{m}^{2}\right.$, day 1$)$ was given to the ICT + CCRT + ACT and ICT + CCRT group patients every three weeks during radiotherapy.

\section{Adjuvant chemotherapy}

Three weeks after finishing CCRT, the ICT + CCRT + ACT group received cisplatin/nedaplatin $\left(75 \sim 80 \mathrm{mg} / \mathrm{m}^{2}\right.$, day 1$)$ and docetaxel $\left(75 \mathrm{mg} / \mathrm{m}^{2}\right.$, day 1$)$ every three weeks for 2 to 5 cycles.

\section{Data analysis}

Medical records from the hospital and telephone counseling were used to collect supplemental data. Following therapy, we reexamined the patients every six months for the first five years and then each year afterward. From the period in which they were initially diagnosed through death or the final follow-up and from diagnosis through the first distant metastasis or the first local-regional recurrence, we computed the overall survival (OS), distant metastasis-free survival (DMFS), locoregional relapse-free survival (LRFS) and disease-free survival (DFS). Version 4.0 of the Common Toxicity Criteria for Adverse Events (CTCAE 4.0) was utilized in the classification of toxicities related to treatment [18]. 
The chi-square test or Fisher's test was used to examine clinical features and adverse occurrences. Parameters such as the number of CCRT and ICT cycles, clinical stage, T stage, $\mathrm{N}$ stage, sex, age and smoking were used as covariables in this study to eliminate potential confounding factors and balance the two groups of clinical characteristics [19]. In addition, we used R software (version 3.6.3) to carry out propensity score matching (PSM).

Survival outcomes of the PSM cohorts in both groups were calculated using the Cox proportional hazards regression model and the Kaplan-Meier technique. Two-sided P-values of $<0.05$ were deemed statistically significant. Hazard ratios (HRs) and 95\% confidence intervals (Cls) were recorded to indicate the prognostic value of the risk factors.

\section{Results}

There were 839 patients classified into the ICT + CCRT and ICT + CCRT + ACT groups, which comprised 443 and 396 patients, respectively. 746 individuals were enrolled in the study after PSM, with 373 patients per group. Table 1 summarizes the clinical features. 
Table 1

Patients' characteristics in the ICT+CCRT and ICT+CCRT+ACT groups before and after PSM in LANC

\begin{tabular}{|c|c|c|c|c|c|c|}
\hline \multirow[t]{2}{*}{ Item } & \multicolumn{3}{|c|}{ Entire cohort (\%) } & \multicolumn{3}{|c|}{ Propensity-score matched cohort (\%) } \\
\hline & ICT + CCRT & ICT + CCRT + ACT & $P$ & ICT + CCRT & ICT + CCRT + ACT & $P$ \\
\hline Total & $443(52.8)$ & $396(47.2)$ & & $373(50.0)$ & $373(50.0)$ & \\
\hline Age (y) & & & 0.284 & & & 0.241 \\
\hline$<46$ & $204(46.0)$ & 197(49.7) & & $168(45.0)$ & 184(49.3) & \\
\hline$\geq 46$ & $239(54.0)$ & 199(50.3) & & $205(55.0)$ & $189(50.7)$ & \\
\hline Gender & & & 0.008 & & & 0.606 \\
\hline Man & $311(70.2)$ & $310(78.3)$ & & 281(75.3) & 287(76.9) & \\
\hline Female & $132(29.8)$ & $86(21.7)$ & & $92(24.7)$ & $86(23.1)$ & \\
\hline T stage & & & 0.508 & & & 0.668 \\
\hline $\mathrm{T} 1-2$ & $101(22.8)$ & $98(24.7)$ & & $87(23.3)$ & $92(24.7)$ & \\
\hline T3-4 & $342(77.2)$ & 298(75.3) & & $286(76.7)$ & $281(75.3)$ & \\
\hline $\mathrm{N}$ stage & & & 0.585 & & & 0.386 \\
\hline No-1 & $142(32.1)$ & $120(30.3)$ & & 123(33.0) & $112(30.0)$ & \\
\hline N2-3 & $301(67.9)$ & $276(69.7)$ & & $250(67.0)$ & $261(70.0)$ & \\
\hline Clinical stage & & & 0.124 & & & 0.592 \\
\hline III & $302(68.2)$ & $250(63.1)$ & & $245(65.7)$ & 238(63.8) & \\
\hline IV & $141(31.8)$ & 146(36.9) & & $128(34.3)$ & $135(36.2)$ & \\
\hline Smoking & & & 0.348 & & & 0.419 \\
\hline No & $257(58.0)$ & $217(54.8)$ & & $176(47.2)$ & $165(44.2)$ & \\
\hline Yes & $186(42.0)$ & $179(45.2)$ & & 197(52.8) & $208(55.8)$ & \\
\hline CCRT cycles & & & 0.234 & & & 0.911 \\
\hline$<2$ & $89(20.1)$ & $93(23.5)$ & & 89 (23.9) & 87 (23.3) & \\
\hline$\geq 2$ & $354(79.9)$ & $303(76.5)$ & & $284(76.1)$ & $286(76.7)$ & \\
\hline IC cycles & & & 0.202 & & & 0.385 \\
\hline$<2$ & $114(25.7)$ & $87(22.0)$ & & $91(24.4)$ & $81(21.7)$ & \\
\hline$\geq 2$ & $329(74.3)$ & $309(78.0)$ & & $282(75.6)$ & 292 (78.3) & \\
\hline Histology & & & 1.000 & & & 1.000 \\
\hline 1 & $2(0.4)$ & $1(0.6)$ & & $2(0.5)$ & $1(0.3)$ & \\
\hline II & $10(2.4)$ & $9(1.8)$ & & $9(2.4)$ & $9(2.4)$ & \\
\hline III & 431(97.2) & 386(97.6) & & 362(97.1) & 363(97.3) & \\
\hline
\end{tabular}


The median follow-up period was 74 months (3-127 months), and The 5-year survival rates for all patients were as follows: OS, 82.9\%; LRFS, 93.0\%; DMFS, 82.6\%; and DFS, 79.2\%. The 5-year OS, DFS, DMFS, and LRFS rates for ICT + CCRT + ACT vs. ICT + CCRT + ACT group were 81.8\% vs. 83.7\% ( $P=0.315), 77.7 \%$ vs. 80.1\% $(P=0.389), 80.6 \%$ vs. $84.4 \%(P=0.185)$, and $92.4 \%$ vs. 93.1\% $(P=0.723)$, respectively (Fig. 2$)$.

Multivariate analysis using Cox regression was performed to account for numerous prognostic variables, as indicated in Table 2. There were no significant changes between the ICT + CCRT and ICT + CCRT + ACT groups in the OS, DFS, DMFS or LRFS rates. The HRs (95\% Cls) of the ICT + CCRT group compared with the ICT + CCRT + ACT group were 1.185 (0.868-1.617), 1.146 (0.663-1.981), $1.299(0.922-1.829)$ and $1.218(0.870-1.705)$, respectively (all $P>0.05)$. Other independent prognostic factors were clinical staging and $\mathrm{N}$ staging (all $P<0.05$ ).

Table 2

Multivariate analyses of prognostic factors in 577 patients with LANC

\begin{tabular}{|c|c|c|c|c|c|c|c|c|}
\hline Variable & os & & DFS & & DMFS & & LRFS & \\
\hline & $\begin{array}{l}\mathrm{HR}(95 \% \\
\mathrm{Cl})\end{array}$ & $P$ & $\begin{array}{l}\mathrm{HR}(95 \% \\
\mathrm{Cl})\end{array}$ & $P$ & $\begin{array}{l}\mathrm{HR}(95 \% \\
\mathrm{Cl})\end{array}$ & $P$ & $\begin{array}{l}\mathrm{HR}(95 \% \\
\mathrm{Cl})\end{array}$ & $P$ \\
\hline $\begin{array}{l}\text { Group (ICT + } \\
\text { CCRT Vs. ICT + } \\
\text { CCRT + ACT })\end{array}$ & $\begin{array}{l}1.218 \\
(0.870 \sim \\
1.705)\end{array}$ & 0.251 & $\begin{array}{l}1.185 \\
(0.868 \sim \\
1.617)\end{array}$ & 0.285 & $\begin{array}{l}1.299 \\
(0.922 \sim \\
1.829)\end{array}$ & 0.135 & $\begin{array}{l}1.146 \\
(0.663 \sim \\
1.981)\end{array}$ & 0.624 \\
\hline $\begin{array}{l}\text { Gender (male } \\
\text { Vs. female) }\end{array}$ & $\begin{array}{l}0.710 \\
(0.473 \sim \\
1.106)\end{array}$ & 0.159 & $\begin{array}{l}0.732 \\
(0.471 \sim \\
1.138)\end{array}$ & 0.166 & $\begin{array}{l}0.650 \\
(0.398 \sim ~ \\
1.061)\end{array}$ & 0.085 & $\begin{array}{l}1.287 \\
(0.577 \sim \\
2.871)\end{array}$ & 0.538 \\
\hline $\begin{array}{l}\text { Age } \\
(<46 V s . \geq 46)\end{array}$ & $\begin{array}{l}1.377 \\
(0.976 \sim \\
1.941)\end{array}$ & 0.068 & $\begin{array}{l}1.183 \\
(0.858 \sim \\
1.630)\end{array}$ & 0.306 & $\begin{array}{l}1.214 \\
(0.852 \sim \\
1.729)\end{array}$ & 0.283 & $\begin{array}{l}1.223 \\
(0.696 \sim \\
2.151)\end{array}$ & 0.484 \\
\hline $\begin{array}{l}\text { Smoking (yes } \\
\text { Vs. no) }\end{array}$ & $\begin{array}{l}0.967 \\
(0.658 \sim \\
1.420)\end{array}$ & 0.864 & $\begin{array}{l}1.003 \\
(0.702 \sim \\
1.433)\end{array}$ & 0.988 & $\begin{array}{l}0.959 \\
(0.651 \sim \\
1.412)\end{array}$ & 0.831 & $\begin{array}{l}1.631 \\
(0.817 \sim \\
3.255)\end{array}$ & 0.165 \\
\hline $\begin{array}{l}\text { T stage }(\mathrm{T} 1-2 \\
\text { Vs. T3-4) }\end{array}$ & $\begin{array}{l}1.302 \\
(0.849 \sim \\
1.994)\end{array}$ & 0.226 & $\begin{array}{l}1.253 \\
(0.849 \sim \\
1.848)\end{array}$ & 0.256 & $\begin{array}{l}1.408 \\
(0.906 \sim \\
2.187)\end{array}$ & 0.128 & $\begin{array}{l}1.379 \\
(0.710 \sim \\
2.679)\end{array}$ & 0.343 \\
\hline $\begin{array}{l}\mathrm{N} \text { stage (N0-1 } \\
\text { Vs. N2-3) }\end{array}$ & $\begin{array}{l}1.604 \\
(1.072 \sim \\
2.400)\end{array}$ & 0.021 & $\begin{array}{l}1.576 \\
(1.080 \sim \\
2.302)\end{array}$ & 0.018 & $\begin{array}{l}1.738 \\
(1.157 \sim \\
2.612)\end{array}$ & 0.008 & $\begin{array}{l}0.814 \\
(0.390 \sim \\
1.700)\end{array}$ & 0.584 \\
\hline $\begin{array}{l}\text { Clinical stage } \\
\text { (III Vs. IV) }\end{array}$ & $\begin{array}{l}2.373 \\
(1.677 \sim \\
3.357)\end{array}$ & 0.001 & $\begin{array}{l}2.346 \\
(1.700 \sim \\
3.238)\end{array}$ & 0.001 & $\begin{array}{l}2.642 \\
(1.852 \sim \\
3.768)\end{array}$ & 0.001 & $\begin{array}{l}1.829 \\
(1.044 \sim \\
3.205)\end{array}$ & 0.035 \\
\hline $\begin{array}{l}\text { IC cycles }(<2 \\
\text { cycles Vs. } \geq 2 \\
\text { cycles) }\end{array}$ & $\begin{array}{l}0.758 \\
(0.518 \sim \\
1.110)\end{array}$ & 0.154 & $\begin{array}{l}0.820 \\
(0.571 \sim \\
1.176)\end{array}$ & 0.281 & $\begin{array}{l}0.694 \\
(0.473 \sim \\
1.019)\end{array}$ & 0.062 & $\begin{array}{l}2.261 \\
(0.960 \sim \\
5.327)\end{array}$ & 0.062 \\
\hline $\begin{array}{l}\text { CCRT cycles (< } \\
2 \text { cycles Vs. } \\
\geq 2 \text { cycles) }\end{array}$ & $\begin{array}{l}0.921(0.624 \\
\sim 1.358)\end{array}$ & 0.677 & $\begin{array}{l}0.861(0.603 \\
\sim 1.231)\end{array}$ & 0.412 & $\begin{array}{l}0.840 \\
(0.568 \sim \\
1.241)\end{array}$ & 0.381 & $\begin{array}{l}1.271(0.649 \\
\sim 2.491)\end{array}$ & 0.484 \\
\hline
\end{tabular}


Prior to PSM, 577 N2-3-positive patients were split into two groups, with 301 patients receiving ICT + CCRT and 276 receiving ICT + CCRT + ACT. There were 474 individuals found after PSM, with 237 patients in each cohort. Table 3 lists the individual baseline characteristics. 
Table 3

Patients' characteristics in the ICT+CCRT and ICT+CCRT+ACT groups before and after PSM in N2-3positive NPC

\begin{tabular}{|c|c|c|c|c|c|c|}
\hline \multirow[t]{2}{*}{ Item } & \multicolumn{3}{|c|}{ Entire cohort (\%) } & \multicolumn{3}{|c|}{ Propensity-score matched cohort (\%) } \\
\hline & ICT+CCRT & ICT+CCRT+ACT & $P$ & ICT+CCRT & ICT+CCRT+ACT & $P$ \\
\hline Total & $301(52.2)$ & $276(47.8)$ & & $237(50.0)$ & $237(50.0)$ & \\
\hline Age & & & 0.815 & & & 0.167 \\
\hline$\nabla 46$ & $147(48.8)$ & $142(51.4)$ & & $135(57.0)$ & $120(50.6)$ & \\
\hline$\geq 46$ & $154(51.2)$ & $134(48.6)$ & & $102(43.0)$ & $117(49.9)$ & \\
\hline Gender & & & 0.045 & & & 0.916 \\
\hline Man & $210(79.4)$ & $213(71.3)$ & & $178(75.1)$ & $177(74.7)$ & \\
\hline Female & $91(20.6)$ & $63(28.7)$ & & $59(24.9)$ & $60(25.3)$ & \\
\hline T stage & & & 0.508 & & & 0.216 \\
\hline T1-2 & $99(32.9)$ & $98(35.5)$ & & $81(34.2)$ & $94(39.7)$ & \\
\hline T3-4 & $202(67.1)$ & 178 (64.5) & & $156(65.8)$ & $143(60.3)$ & \\
\hline N stage & & & 0.359 & & & 0.910 \\
\hline N 2 & 235 (78.1) & 224 (81.2) & & $187(78.9)$ & 188 (79.3) & \\
\hline N 3 & 66 (21.9) & $52(18.8)$ & & $50(21.1)$ & $49(20.7)$ & \\
\hline Clinical stage & & & 0.383 & & & 0.558 \\
\hline III & $197(65.4)$ & $171(62.0)$ & & $156(65.8)$ & $162(68.4)$ & \\
\hline IV & 104 (34.6) & $105(38.0)$ & & $81(34.2)$ & 75 (31.6) & \\
\hline Smoking & & & 0.300 & & & 0.645 \\
\hline No & $170(56.5)$ & $144(52.2)$ & & $132(55.7)$ & $127(53.6)$ & \\
\hline Yes & $131(43.5)$ & 132 (47.8) & & $105(44.3)$ & $110(46.4)$ & \\
\hline CCRT cycles & & & 0.642 & & & 0.514 \\
\hline$\bowtie 2$ & 66 (21.9) & 65 (23.6) & & $58(24.5)$ & $52(21.9)$ & \\
\hline$\geq 2$ & 235 (78.1) & 211(76.4) & & $179(75.5)$ & 185 (78.1) & \\
\hline IC cycles & & & 0.451 & & & 0.230 \\
\hline$\otimes 2$ & $70(23.3)$ & $57(20.7)$ & & $60(25.3)$ & $49(20.7)$ & \\
\hline$\geq 2$ & 231 (76.7) & 219 (79.3) & & $177(74.7)$ & 188 (79.3) & \\
\hline Histology & & & 0.891 & & & 0.886 \\
\hline I & $1(0.3)$ & $1(0.4)$ & & $1(0.4)$ & $1(0.4)$ & \\
\hline ॥ & $6(2.0)$ & $7(2.5)$ & & $5(2.1)$ & $7(3.0)$ & \\
\hline
\end{tabular}




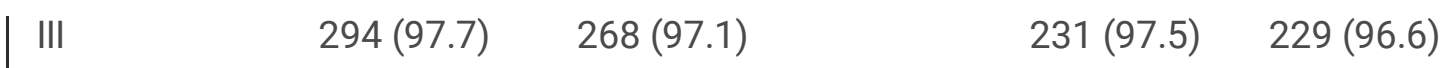

The median follow-up for the entire cohort was 74 months (3-127 months). The 5-year LRFS, OS, DFS and DMFS rates for the ICT + CCRT and ICT + CCRT + ACT groups were $90.8 \%$ vs. $94.7 \%(P=0.177), 73.4 \%$ vs. $81.7 \%(P=$ $0.034), 78.9 \%$ vs. $85.0 \%(P=0.029)$ and $76.0 \%$ vs. $84.9 \%(P=0.019)$, respectively (Fig. 3).

Multivariate analysis revealed that ICT + CCRT + ACT resulted in a substantial improvement in 5-year DMFS (HR: 1.724; $95 \% \mathrm{Cl}: 1.123-2.648 ; P=0.013)$, DFS (HR: $1.565 ; 95 \% \mathrm{Cl}: 1.055-2.323 ; P=0.025)$, LRFS (HR: $1.638 ; 95 \% \mathrm{Cl}$ : $0.826-3.246 ; P=0.158$ ) and OS (HR: $1.064 ; 95 \% \mathrm{Cl}: 1.059-2.430 ; P=0.026)$. Clinical staging, $\mathrm{N}$ staging, and ICT cycles were all independent variables for OS, DFS, and DMFS, as shown in Table 4 (all $P<0.05$ ). 
Table 4

Multivariate analyses of prognostic factors in patients with N2-3-positive LANC

\begin{tabular}{|c|c|c|c|c|c|c|c|c|}
\hline Variable & OS & & DFS & & DMFS & & LRRFS & \\
\hline & $\begin{array}{l}\mathrm{HR}(95 \% \\
\mathrm{Cl})\end{array}$ & $P$ & $\begin{array}{l}\mathrm{HR}(95 \% \\
\mathrm{Cl})\end{array}$ & $P$ & $\begin{array}{l}\mathrm{HR}(95 \% \\
\mathrm{Cl})\end{array}$ & $P$ & $\begin{array}{l}\text { HR } \\
(95 \% \\
\mathrm{Cl})\end{array}$ & $P$ \\
\hline $\begin{array}{l}\text { Group (ICT + CCRT } \\
\text { Vs. ICT + CCRT + ACT) }\end{array}$ & $\begin{array}{l}1.604 \\
(1.059 \sim \\
2.430)\end{array}$ & 0.026 & $\begin{array}{l}1.565 \\
(1.055 \sim \\
2.323)\end{array}$ & 0.025 & $\begin{array}{l}1.724 \\
(1.123 \sim \\
2.648)\end{array}$ & 0.013 & $\begin{array}{l}1.638 \\
(0.826 \\
\sim \\
3.246)\end{array}$ & 0.158 \\
\hline $\begin{array}{l}\text { Gender (male Vs. } \\
\text { female) }\end{array}$ & $\begin{array}{l}0.735 \\
(0.413 \sim \\
1.307)\end{array}$ & 0.294 & $\begin{array}{l}0.834 \\
(0.478 \sim \\
1.454)\end{array}$ & 0.522 & $\begin{array}{l}0.700 \\
(0.385 \sim \\
1.273)\end{array}$ & 0.243 & $\begin{array}{l}2.061 \\
(0.716 \\
\sim \\
5.935)\end{array}$ & 0.180 \\
\hline $\operatorname{Age}(<46 V s . \geq 46)$ & $\begin{array}{l}1.036 \\
(0.682 \sim \\
1.575)\end{array}$ & 0.867 & $\begin{array}{l}0.884 \\
(0.591 \sim \\
1.321)\end{array}$ & 0.547 & $\begin{array}{l}0.947 \\
(0.615 \sim \\
1.459)\end{array}$ & 0.806 & $\begin{array}{l}0.989 \\
(0.494 \\
\sim \\
1.978)\end{array}$ & 0.975 \\
\hline Smoking (yes Vs. no) & $\begin{array}{l}1.181 \\
(0.734 \sim \\
1.901)\end{array}$ & 0.492 & $\begin{array}{l}1.379 \\
(0.873 \sim \\
2.179)\end{array}$ & 0.168 & $\begin{array}{l}1.211 \\
(0.751 \sim \\
1.953)\end{array}$ & 0.433 & $\begin{array}{l}2.700 \\
(1.035 \\
\sim \\
7.041)\end{array}$ & 0.042 \\
\hline $\begin{array}{l}\text { T stage (T1-2 Vs. } \\
\text { T3-4) }\end{array}$ & $\begin{array}{l}1.102 \\
(0.598 \sim \\
2.031)\end{array}$ & 0.755 & $\begin{array}{l}1.076 \\
(0.602 \sim \\
1.924)\end{array}$ & 0.805 & $\begin{array}{l}1.135 \\
(0.614 \sim \\
2.099)\end{array}$ & 0.685 & $\begin{array}{l}0.572 \\
(0.199 \\
\tilde{1} \\
1.642)\end{array}$ & 0.299 \\
\hline $\begin{array}{l}\mathrm{N} \text { stage (N0-1 Vs. } \\
\mathrm{N} 2-3)\end{array}$ & $\begin{array}{l}1.964 \\
(1.232 \sim \\
3.131)\end{array}$ & 0.005 & $\begin{array}{l}1.852 \\
(1.189 \sim \\
2.883)\end{array}$ & 0.006 & $\begin{array}{l}2.290 \\
(1.415 \sim \\
3.703)\end{array}$ & 0.001 & $\begin{array}{l}0.750 \\
(0.330 \\
\sim \\
1.701)\end{array}$ & 0.491 \\
\hline $\begin{array}{l}\text { Clinical stage (III Vs. } \\
\text { IV) }\end{array}$ & $\begin{array}{l}3.374 \\
(1.783 \sim \\
6.384)\end{array}$ & 0.001 & $\begin{array}{l}3.078 \\
(1.697 \sim \\
5.575)\end{array}$ & 0.001 & $\begin{array}{l}4.057 \\
(2.102 \sim \\
7.833)\end{array}$ & 0.001 & $\begin{array}{l}1.954 \\
(0.791 \\
\sim \\
4.828)\end{array}$ & 0.147 \\
\hline $\begin{array}{l}\text { IC cycles }(<2 \text { cycles } \\
\text { Vs. } \geq 2 \text { cycles })\end{array}$ & $\begin{array}{l}0.608 \\
(0.388 \sim \\
0.953)\end{array}$ & 0.030 & $\begin{array}{l}0.609 \\
(0.396 \sim \\
0.934)\end{array}$ & 0.023 & $\begin{array}{l}0.592 \\
(0.374 \sim \\
0.937)\end{array}$ & 0.025 & $\begin{array}{l}0.838 \\
(0.391 \\
\tilde{1} \\
1.793)\end{array}$ & 0.648 \\
\hline $\begin{array}{l}\text { CCRT cycles }(<2 \\
\text { cycles Vs. } \geq 2 \text { cycles) }\end{array}$ & $\begin{array}{l}1.006 \\
(0.619 \sim \\
1.635)\end{array}$ & 0.980 & $\begin{array}{l}1.048 \\
(0.653 \sim \\
1.684)\end{array}$ & 0.845 & $\begin{array}{l}1.026 \\
(0.618 \sim \\
1.704)\end{array}$ & 0.921 & $\begin{array}{l}1.336 \\
(0.569 \\
\sim \\
3.136)\end{array}$ & 0.506 \\
\hline
\end{tabular}

Toxic effects

We compared the treatment-related toxicity of the ICT + CCRT and ICT + CCRT + ACT groups, and the findings are presented in Table 5. The incidence of nonhematologic toxicities did not differ significantly between the two groups (all $P>0.05$ ). Compared to ICT + CCRT, ICT + CCRT + ACT substantially increased the prevalence of grade $3 / 4$ leukocytopenia $(P<0.001)$ and neutropenia $(P<0.001)$. 
Table 5

Adverse events

\begin{tabular}{|c|c|c|c|c|c|c|c|c|c|c|}
\hline \multirow{3}{*}{ Adverse event } & \multicolumn{5}{|c|}{$\begin{array}{l}\text { after propensity score matching in patients } \\
\text { with LANC }\end{array}$} & \multicolumn{5}{|c|}{$\begin{array}{l}\text { after propensity score matching in patients } \\
\text { with N2-3-positive LANC }\end{array}$} \\
\hline & \multicolumn{2}{|c|}{$\begin{array}{l}\text { ICT + } \\
\text { CCRT (case\%) }\end{array}$} & \multicolumn{2}{|c|}{$\begin{array}{l}\text { ICT + CCRT + } \\
\text { ACT (case } \%)\end{array}$} & \multirow[t]{2}{*}{$\begin{array}{l}P \text { - } \\
\text { value }\end{array}$} & \multicolumn{2}{|c|}{$\begin{array}{l}\text { ICT + } \\
\text { CCRT (case\%) }\end{array}$} & \multicolumn{2}{|c|}{$\begin{array}{l}\text { ICT + CCRT + } \\
\text { ACT (case } \%)\end{array}$} & \multirow[t]{2}{*}{$\begin{array}{l}P \text { - } \\
\text { value }\end{array}$} \\
\hline & $\begin{array}{l}\text { Grade } \\
0-2\end{array}$ & $\begin{array}{l}\text { Grade } \\
3-4\end{array}$ & $\begin{array}{l}\text { Grade } \\
0-2\end{array}$ & $\begin{array}{l}\text { Grade } \\
3-4\end{array}$ & & $\begin{array}{l}\text { Grade } \\
0-2\end{array}$ & $\begin{array}{l}\text { Grade } \\
3-4\end{array}$ & $\begin{array}{l}\text { Grade } \\
0-2\end{array}$ & $\begin{array}{l}\text { Grade } \\
3-4\end{array}$ & \\
\hline Leukocytopenia & $\begin{array}{l}285 \\
(76.4)\end{array}$ & $\begin{array}{l}88 \\
(23.6)\end{array}$ & $\begin{array}{l}196 \\
(52.5)\end{array}$ & $\begin{array}{l}177 \\
(47.5)\end{array}$ & $<0.001$ & $\begin{array}{l}168 \\
(70.9)\end{array}$ & $\begin{array}{l}69 \\
(29.1)\end{array}$ & $\begin{array}{l}131 \\
(55.3)\end{array}$ & $\begin{array}{l}106 \\
(44.7)\end{array}$ & $\begin{array}{l}< \\
0.001\end{array}$ \\
\hline Neutropenia & $\begin{array}{l}294 \\
(78.8)\end{array}$ & $\begin{array}{l}79 \\
(21.2)\end{array}$ & $\begin{array}{l}234 \\
(62.7)\end{array}$ & $\begin{array}{l}139 \\
(31.3)\end{array}$ & $\begin{array}{l}< \\
0.001\end{array}$ & $\begin{array}{l}182 \\
(76.8)\end{array}$ & $\begin{array}{l}55 \\
(23.2)\end{array}$ & $\begin{array}{l}153 \\
(64.6)\end{array}$ & $\begin{array}{l}84 \\
(35.4)\end{array}$ & 0.003 \\
\hline Thrombocytopenia & $\begin{array}{l}358 \\
(96.0)\end{array}$ & $\begin{array}{l}15 \\
(4.0)\end{array}$ & $\begin{array}{l}360 \\
(96.5)\end{array}$ & $\begin{array}{l}13 \\
(3.5)\end{array}$ & 0.700 & $\begin{array}{l}227 \\
(95.8)\end{array}$ & $\begin{array}{l}10 \\
(4.2)\end{array}$ & $\begin{array}{l}232 \\
(97.9)\end{array}$ & $\begin{array}{l}5 \\
(2.1)\end{array}$ & 0.294 \\
\hline Anemia & $\begin{array}{l}355 \\
(95.2)\end{array}$ & $\begin{array}{l}18 \\
(4.8)\end{array}$ & $\begin{array}{l}351 \\
(94.1)\end{array}$ & $\begin{array}{l}22 \\
(5.9)\end{array}$ & 0.516 & $\begin{array}{l}222 \\
(93.7)\end{array}$ & $\begin{array}{l}15 \\
(6.3)\end{array}$ & $\begin{array}{l}225 \\
(94.9)\end{array}$ & $\begin{array}{l}12 \\
(5.1)\end{array}$ & 0.552 \\
\hline \multicolumn{11}{|l|}{ Non-Hematologic } \\
\hline Liver function & $\begin{array}{l}367 \\
(98.4)\end{array}$ & $\begin{array}{l}6 \\
(1.6)\end{array}$ & $\begin{array}{l}368 \\
(98.7)\end{array}$ & $\begin{array}{l}5 \\
(1.3)\end{array}$ & 0.761 & $\begin{array}{l}233 \\
(98.3)\end{array}$ & $\begin{array}{l}4 \\
(1.7)\end{array}$ & $\begin{array}{l}235 \\
(99.2)\end{array}$ & $\begin{array}{l}2 \\
(0.8)\end{array}$ & 0.686 \\
\hline Renal function & $\begin{array}{l}373 \\
(100)\end{array}$ & $0(0)$ & $\begin{array}{l}373 \\
(100)\end{array}$ & $0(0)$ & 1.000 & $\begin{array}{l}237 \\
(100)\end{array}$ & $0(0)$ & $\begin{array}{l}237 \\
(100)\end{array}$ & $0(0)$ & 1.000 \\
\hline Oral mucositis & $\begin{array}{l}290 \\
(77.7)\end{array}$ & $\begin{array}{l}83 \\
(22.3)\end{array}$ & $\begin{array}{l}300 \\
(80.4)\end{array}$ & $\begin{array}{l}73 \\
(19.6)\end{array}$ & 0.368 & $\begin{array}{l}176 \\
(74.3)\end{array}$ & $\begin{array}{l}61 \\
(25.7)\end{array}$ & $\begin{array}{l}188 \\
(79.3)\end{array}$ & $\begin{array}{l}49 \\
(20.7)\end{array}$ & 0.192 \\
\hline Nausea/vomiting & $\begin{array}{l}352 \\
(94.4)\end{array}$ & $\begin{array}{l}21 \\
(5.6)\end{array}$ & $\begin{array}{l}354 \\
(94.9)\end{array}$ & $\begin{array}{l}19 \\
(5.1)\end{array}$ & 0.745 & $\begin{array}{l}224 \\
(94.5)\end{array}$ & $\begin{array}{l}13 \\
(5.5)\end{array}$ & $\begin{array}{l}229 \\
(96.6)\end{array}$ & $\begin{array}{l}8 \\
(3.4)\end{array}$ & 0.264 \\
\hline
\end{tabular}

\section{Discussion}

This study is first to examine the clinical effectiveness of ICT + CCRT followed by ACT in LANC patients. We discovered that ACT following ICT + CCRT had no significant effect on improving the survival of LANC patients. Furthermore, we observed that patients with N2-3-positive LANC in the ICT + CCRT + ACT group had better 5-yesr OS, DFS, and DMFS than those in the ICT + CCRT group. Based on this study, we suggest that patients with N2-3positive LANC should be treated with the ICT + CCRT + ACT regimen to further reduce the incidence of distant metastasis.

CCRT is the cornerstone of the treatment of $\operatorname{LANC}[5,20]$. With the wide application of IMRT, the incidence of local control of NPC has been significantly improved; however, the occurrence of distant metastasis has become the main cause of treatment failure[21, 22]. In recent years, the combination of ICT or ACT with CCRT has minimized the incidence of distant metastases in LANC patients [23]. Compared with CCRT alone, CCRT following ICT was found to improve 5 -year OS in an IPD pooled analysis ( $\mathrm{HR}=0.75,95 \% \mathrm{Cl} 0.57 \sim 0.99)$ by improving DMFS (HR $=0.68,95 \%$ $\mathrm{Cl} 0.90 \sim 0.51$ ) [8]. Since then, IC + CCRT has become the standard treatment regimen for LANC according to 2018 NCCN guidelines [7]. Although the addition of ICT to CCRT can improve the survival of patients with LANC, a small number of patients with a high risk of metastasis still have a residual primary tumor and lymph node metastasis after treatment. ACT is administered to reduce residual tumor cells, prevent relapse, and eliminate micrometastases 
[24]. We found that the ICT + CCRT and CCRT + ACT regimens had the same level of evidence, both of which were 2A [7]. Therefore, whether ACT following ICT plus CCRT can further improve survival in patients with high-risk LANC is worth further exploration.

ACT remains controversial because a previous study failed to demonstrate clinical effectiveness [25-27]. Chen et al showed that there were no significant differences between the CCRT group and the CCRT + ACT group in 5-year FFS (75\% vs. 71\%; $P=0.45)$, OS (83\% vs. 80\%; $P=0.35)$, DMFS (85\% vs. 80\%; $P=0.30)$ and LRFS $(91 \%$ vs. $90 \% ; P=0.73)$. The main reason for this was that most patients with poor tolerance after CCRT exhibited decreased compliance. Approximately $40 \%$ of patients did not complete three cycles of ACT, and 44 of them refused ACT $[25,26]$. In this study, all the patients in the ICT + CCRT + ACT group received two or more courses of ACT, providing more reliable results.

Additionally, not all patients are suitable for ACT, and patients who are more likely to benefit from ACT are still uncertain at present. Many N0-1-positive patients were included in Chen et al.'s study. Similarly, Anthony et al. [27] explored the connection between ACT and plasma Epstein-Barr virus DNA. This study suggested that ACT with cisplatin and gemcitabine (GP) did not improve 5-year DMFS. Therefore, using biomarkers cannot screen patients who can benefit from ACT. As per our findings, the ICT + CCRT + ACT regimen could further improve the OS, DFS, and DMFS in N2-3-positive LANC patients. The 5-year OS, DFS and DMFS rates in the ICT + CCRT group were 78.9\%, $73.4 \%$ and $76.0 \%$, respectively, which were significantly lower than those in the ICT + CCRT + ACT group (85.0\%, $81.7 \%$ and $84.9 \%$, respectively) (all $P<0.05$ ). Therefore, according to the results of this study, we believe that the addition of ACT based on the IC + CCRT regimen is suitable for those with N2-3-positive LANC.

Generally, cisplatin plus fluorouracil (PF) is the most common ACT regimen and is the preferred treatment recommended by the guidelines. However, ACT with the PF regimen may not be the most effective, and some new chemotherapy agents (such as capecitabine, docetaxel, and capecitabine) may further improve the clinical value of ACT. A recent study [25] found that ACT with capecitabine could improve the 3-year FFS in LANC patients. Zhang et al. reported that ACT with the TP regimen is an effective strategy for treating LANC. Likewise, our study demonstrates the clinical value and feasibility of ACT with the TP regimen.

Docetaxel is widely used in head and neck squamous cell carcinoma because it impairs mitosis and induces apoptosis[28]. Meanwhile, docetaxel has demonstrated a lower rate of grade 3 and 4 nausea/vomiting than fluorouracil. A retrospective study revealed that compared with TP regimen, ICT with 5-fluorouracil-based regimens significantly increased the incidence of mucositis/vomiting toxicity [29]. Zhang et al. [30] discovered that ACT with the TP regimen is a successful treatment for LANC, with no patients experiencing grade 3/4 nausea or vomiting. Therefore, LANC patients tolerate the TP regimen better than the PF regimen, and $75 \%$ of the patients in the current study completed three cycles of ACT.

According to Zou et al., the rate of grade 3/4 leukocytopenia and neutropenia was significantly elevated, and the incidence was $67.5 \%$ and $55.8 \%$, respectively, which was similar to the result of our study. We found that grade $3 / 4$ leukocytopenia/neutropenia in the ICT + CCRT + ACT group was significantly greater than that in the ICT + CCRT group (all $P<0.001$ ). Additionally, our study showed no notable differences in $3 / 4$ nausea/vomiting and oral mucositis between the two groups, which was in line with previous research [30].

There are some limitations to the current study. First, this is a retrospective study using data from a single center. Another caveat is that some biases were inevitable since it was retrospective. PSM and multivariable regression can help us minimize confounding effects, but other unmeasured potential confounding factors may still exist. 
Meanwhile, some outpatient information was not fully documented. Therefore, large prospective clinical studies will be required to confirm our results.

\section{Conclusion}

In summary, our research revealed no notable differences in OS, DFS, DMFS, and LRFS between the ICT + CCRT and ICT + CCRT + ACT groups of LANC patients. When we excluded N0-1-positive patients and performed PSM in the ICT + CCRT and ICT + CCRT + ACT groups, we found that the ICT + CCRT + ACT regimen could improve the DMFS in N2-

3-positive LANC patients and prolong the OS. Moreover, adding ACT after ICT plus CCRT increased the incidence of adverse effects, such as grade 3/4 leukocytopenia and neutropenia, but these toxic events are controllable in the clinic. Our studies indicated the efficacy and feasibility of the ICT + CCRT + ACT regimen, so we recommend that patients with N2-3-positive LANC should be treated with the ICT + CCRT + ACT regimen.

\section{Declarations}

\section{Author contributions}

Author contributions: Rong-Hui Zheng, Ya-Wei Yuan and Hao-Yun Tao conceived the study. Hui Liu, Cai-Xian He, Ran $\mathrm{Li}$ and Hao-Yun Tao collected all clinical data. Hao-Yun Tao and Ran Li performed the statistical analyses. Hao-Yun Tao and Kun-Peng Du prepared and edited the manuscript. All authors read and approved the final manuscript.

\section{Funding}

There was no external funding to support the writing of this article.

\section{Ethics approval}

This retrospective study was approved by the Institutional Review Board (IRB) of the Affiliated Cancer Hospital \& Institute of Guangzhou Medical University. Patients were required to provide written informed consent before enrolling in the study.

\section{Competing Interests}

The authors declare they have no competing interests.

\section{Availability of data and materials}

The datasets generated during and/or analyzed during the current study are available in the Figshare repository: https://figshare.com/s/534685768da5c9320487

\section{References}

1. Chen Y-P, Chan ATC, Le Q-T, Blanchard P, Sun Y, Ma J. Nasopharyngeal carcinoma. The Lancet. 2019;394(10192):64-80. doi: 10.1016/s0140-6736(19)30956-0.

2. Afqir S, Ismaili N, Errihani H. Concurrent chemoradiotherapy in the management of advanced nasopharyngeal carcinoma: current status. J Cancer Res Ther. 2009;5(1):3-7. Epub 2009/03/19. doi: 10.4103/0973-1482.48763. 
3. Goto Y, Kodaira T, Fuwa N, Mizoguchi N, Nakahara R, Nomura M, et al. Alternating chemoradiotherapy in patients with nasopharyngeal cancer: prognostic factors and proposal for individualization of therapy. J Radiat Res. 2013;54(1):98-107. Epub 2012/08/28. doi: 10.1093/jrr/rrs071.

4. Al-Sarraf M, LeBlanc M, Giri PG, Fu KK, Cooper J, Vuong T, et al. Chemoradiotherapy versus radiotherapy in patients with advanced nasopharyngeal cancer: phase III randomized Intergroup study 0099. J Clin Oncol 1998, 16(4):1310-1317.

5. Lin JC, Jan JS, Hsu CY, Liang WM, Jiang RS, Wang WY. Phase III study of concurrent chemoradiotherapy versus radiotherapy alone for advanced nasopharyngeal carcinoma: positive effect on overall and progression-free survival. J Clin Oncol. 2003;21(4):631-7. Epub 2003/02/15. doi: 10.1200/JC0.2003.06.158.

6. Chen QY, Wen YF, Guo L, Liu H, Huang PY, Mo HY, et al. Concurrent chemoradiotherapy vs radiotherapy alone in stage II nasopharyngeal carcinoma: phase III randomized trial. J Natl Cancer Inst. 2011;103(23):1761-70. Epub 2011/11/08. doi: 10.1093/jnci/djr432.

7. National Comprehensive Cancer Network for Head and Neck Cancers. Version 1. 2018.

8. Chen YP, Tang LL, Yang Q, Poh SS, Hui EP, Chan ATC, et al. Induction Chemotherapy plus Concurrent Chemoradiotherapy in Endemic Nasopharyngeal Carcinoma: Individual Patient Data Pooled Analysis of Four Randomized Trials. Clin Cancer Res. 2018;24(8):1824-33. Epub 2018/02/13. doi: 10.1158/1078-0432.CCR-172656.

9. Hui EP, Ma BB, Leung SF, King AD, Mo F, Kam MK, et al. Randomized phase II trial of concurrent cisplatinradiotherapy with or without neoadjuvant docetaxel and cisplatin in advanced nasopharyngeal carcinoma. J Clin Oncol. 2009;27(2):242-9. Epub 2008/12/10. doi: 10.1200/JC0.2008.18.1545.

10. Sun Y, Li W-F, Chen N-Y, Zhang N, Hu G-Q, Xie F-Y, et al. Induction chemotherapy plus concurrent chemoradiotherapy versus concurrent chemoradiotherapy alone in locoregionally advanced nasopharyngeal carcinoma: a phase 3, multicentre, randomised controlled trial. The Lancet Oncology. 2016;17(11):1509-20. doi: 10.1016/s1470-2045(16)30410-7.

11. Li WF, Chen NY, Zhang N, Hu GQ, Xie FY, Sun Y, et al. Concurrent chemoradiotherapy with/without induction chemotherapy in locoregionally advanced nasopharyngeal carcinoma: Long-term results of phase 3 randomized controlled trial. International Journal of Cancer. 2019;145(1):295-305. doi: 10.1002/ijc.32099.

12. Zhang Y, Chen L, Hu GQ, Zhang N, Zhu XD, Yang KY, et al. Gemcitabine and Cisplatin Induction Chemotherapy in Nasopharyngeal Carcinoma. N Engl J Med. 2019;381(12):1124-35. Epub 2019/06/01. doi: 10.1056/NEJMoa1905287.

13. Rusch VW, Giroux DJ, Kraut MJ, Crowley J, Hazuka M, Winton T, et al. Induction chemoradiation and surgical resection for superior sulcus non-small-cell lung carcinomas: Iong-term results of Southwest Oncology Group Trial 9416 (Intergroup Trial 0160). J Clin Oncol. 2007;25(3):313-8. Epub 2007/01/20. doi: 10.1200/JC0.2006.08.2826.

14. White AA, Lee DN, Mazzola E, Kucukak S, Polhemus E, Jaklitsch MT, et al. Adjuvant therapy following induction therapy and surgery improves survival in N2-positive non-small cell lung cancer. J Surg Oncol. 2021;123(2):57986. Epub 2020/12/02. doi: 10.1002/jso.26305.

15. Kwong KF, Edelman MJ, Suntharalingam M, Cooper LB, Gamliel Z, Burrows W, et al. High-dose radiotherapy in trimodality treatment of Pancoast tumors results in high pathologic complete response rates and excellent long-term survival. J Thorac Cardiovasc Surg. 2005;129(6):1250-7. Epub 2005/06/09. doi: 10.1016/j.jtcvs.2004.12.050.

Page $14 / 18$ 
16. Ribassin-Majed L, Marguet S, Lee AWM, Ng WT, Ma J, Chan ATC, et al. What Is the Best Treatment of Locally Advanced Nasopharyngeal Carcinoma? An Individual Patient Data Network Meta-Analysis. J Clin Oncol. 2017;35(5):498-505. Epub 2016/12/06. doi: 10.1200/JC0.2016.67.4119.

17. Zou R, Yuan JJ, Li Q, Ding JW, Liao B, Tu ZW, et al. The Clinical Outcomes and Toxicities of Induction Chemotherapy Followed by Concurrent Chemoradiotherapy Plus Adjuvant Chemotherapy in Locoregionally Advanced Nasopharyngeal Carcinoma. Front Oncol. 2020;10:619625. Epub 2021/04/02. doi: 10.3389/fonc. 2020.619625 .

18. National Cancer Institute-Common Toxicity Criteria Adverse Events Versions 4. http://evs.nci.nih.gov/ftp1/CTCAE/ CTCAE_4.03_2010-06 14_QuickReference_8.5x11.pdf.

19. Austin PC. The performance of different propensity score methods for estimating marginal hazard ratios. Stat Med. 2013;32(16):2837-49. Epub 2012/12/15. doi: 10.1002/sim.5705.

20. Chan AT, Leung SF, Ngan RK, Teo PM, Lau WH, Kwan WH, et al. Overall survival after concurrent cisplatinradiotherapy compared with radiotherapy alone in locoregionally advanced nasopharyngeal carcinoma. J Natl Cancer Inst. 2005;97(7):536-9. Epub 2005/04/07. doi: 10.1093/jnci/dji084.

21. Sun X, Su S, Chen C, Han F, Zhao C, Xiao W, et al. Long-term outcomes of intensity-modulated radiotherapy for 868 patients with nasopharyngeal carcinoma: an analysis of survival and treatment toxicities. Radiother Oncol. 2014;110(3):398-403. Epub 2013/11/16. doi: 10.1016/j.radonc.2013.10.020.

22. Tang L-Q, Chen Q-Y, Fan W, Liu H, Zhang L, Guo L, et al. Prospective Study of Tailoring Whole-Body DualModality [18F]Fluorodeoxyglucose Positron Emission Tomography/Computed Tomography With Plasma Epstein-Barr Virus DNA for Detecting Distant Metastasis in Endemic Nasopharyngeal Carcinoma at Initial Staging. Journal of Clinical Oncology. 2013;31(23):2861-9. doi: 10.1200/jco.2012.46.0816.

23. Yu-Pei Chen, Nofisat Ismaila, Melvin L K Chua, A Dimitrios Colevas, Robert Haddad, Shao Hui Huang. et al. Chemotherapy in Combination With Radiotherapy for Definitive-Intent Treatment of Stage II-IVA Nasopharyngeal Carcinoma: CSCO and ASCO Guideline 2021 Mar 1;39(7):840-859.doi: 10.1200/JC0.20.03237.

24. Song L, Liu H, Ma L, Zhang X, Jiang Z, Jiang C. Inhibition of autophagy by 3-MA enhances endoplasmic reticulum stress-induced apoptosis in human nasopharyngeal carcinoma cells. Oncol Lett. 2013;6(4):1031-8. Epub 2013/10/19. doi: 10.3892/ol.2013.1498.

25. Chen L, Hu C-S, Chen X-Z, Hu G-Q, Cheng Z-B, Sun Y, et al. Concurrent chemoradiotherapy plus adjuvant chemotherapy versus concurrent chemoradiotherapy alone in patients with locoregionally advanced nasopharyngeal carcinoma: a phase 3 multicentre randomised controlled trial. The Lancet Oncology. 2012;13(2):163-71. doi: 10.1016/s1470-2045(11)70320-5.

26. Chen L, Hu CS, Chen XZ, Hu GQ, Cheng ZB, Sun Y, et al. Adjuvant chemotherapy in patients with locoregionally advanced nasopharyngeal carcinoma: Long-term results of a phase 3 multicentre randomised controlled trial. Eur J Cancer. 2017;75:150-8. Epub 2017/02/27. doi: 10.1016/j.ejca.2017.01.002.

27. Anthony T C Chan, Edwin P Hui, Roger K C Ngan, Stewart Y Tung, Ashley C K Cheng, Wai T Ng,et al. Analysis of Plasma Epstein-Barr Virus DNA in Nasopharyngeal Cancer After Chemoradiation to Identify High-Risk Patients for Adjuvant Chemotherapy: A Randomized Controlled Trial.2018 Jul 10.doi: 10.1200/JC0.2018.77.7847.

28. Kim E, Matsuse M, Saenko V, Suzuki K, Ohtsuru A, Mitsutake N, et al. Imatinib enhances docetaxel-induced apoptosis through inhibition of nuclear factor-kappaB activation in anaplastic thyroid carcinoma cells. Thyroid. 2012;22(7):717-24. Epub 2012/06/02. doi: 10.1089/thy.2011.0380.

29. He Y, Guo T, Wang J, Sun Y, Guan H, Wu S, et al. Which induction chemotherapy regimen followed by cisplatinbased concurrent chemoradiotherapy is the best choice among PF, TP and TPF for locoregionally advanced 
nasopharyngeal carcinoma? Ann Transl Med. 2019;7(5):104. Epub 2019/04/26. doi:

10.21037/atm.2019.02.15.

30. Zhang X, Du L, Zhao F, Wang Q, Yang S, Ma L. A Phase II Clinical Trial of Concurrent Helical Tomotherapy plus Cetuximab Followed by Adjuvant Chemotherapy with Cisplatin and Docetaxel for Locally Advanced Nasopharyngeal Carcinoma. Int J Biol Sci. 2016;12(4):446-53. Epub 2016/03/29. doi: 10.7150/ijbs.12937.

\section{Figures}

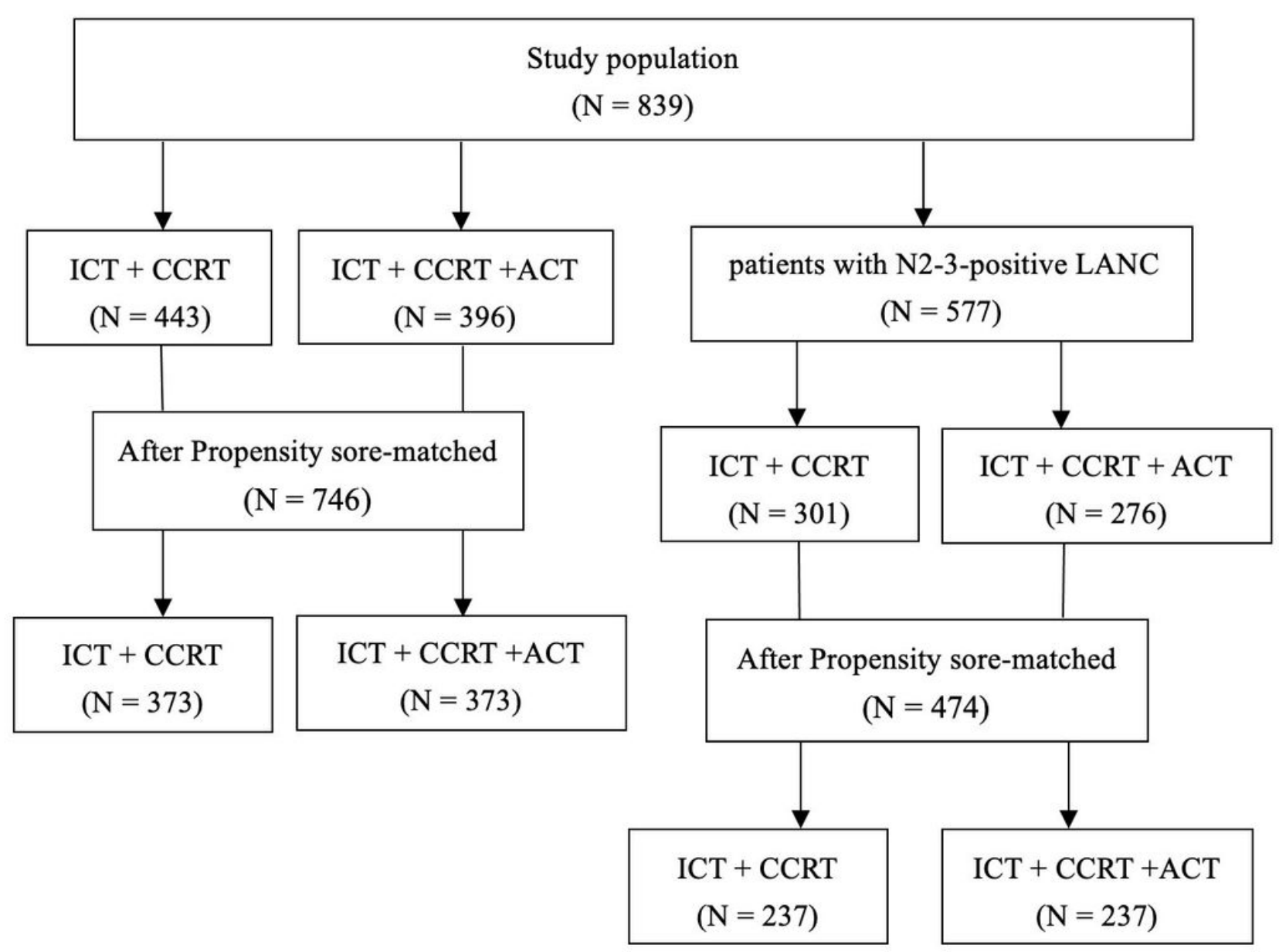

Fig. 1 Flow chart of patient selection. IC: Induction Chemotherapy; CCRT: Concurrent Chemoradiotherapy; AC: Adjuvant Chemotherapy

Figure 1

See image above for figure legend. 
A

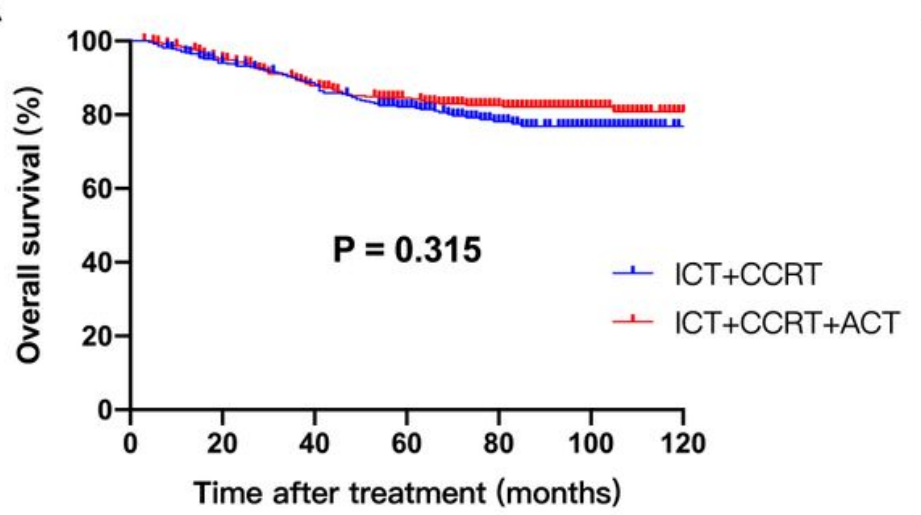

C

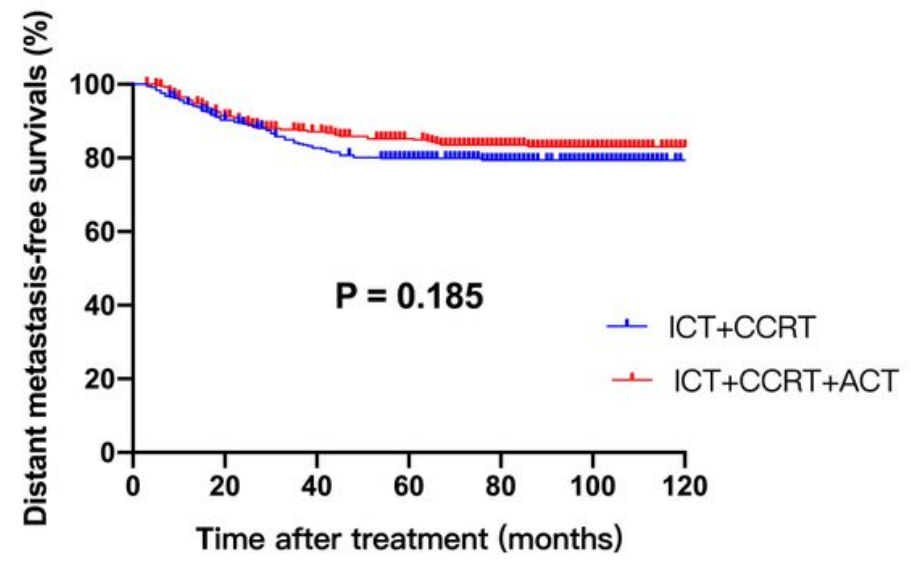

B

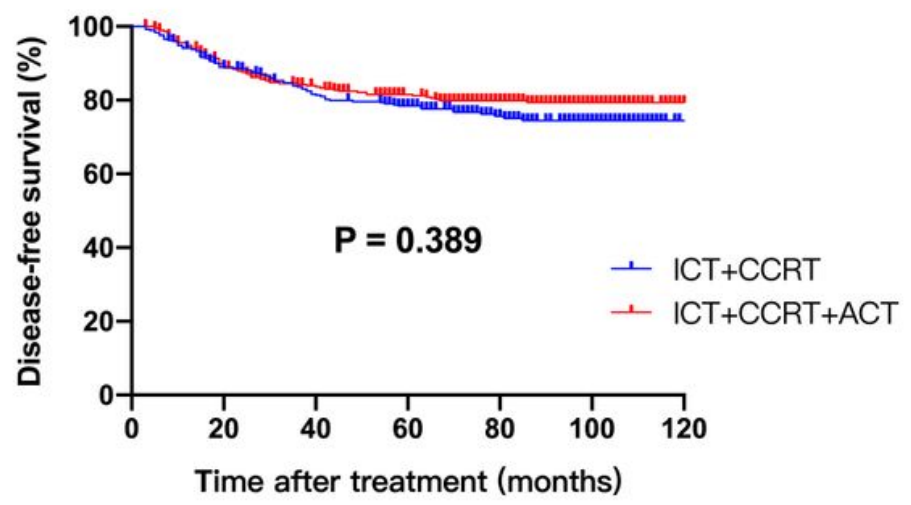

D

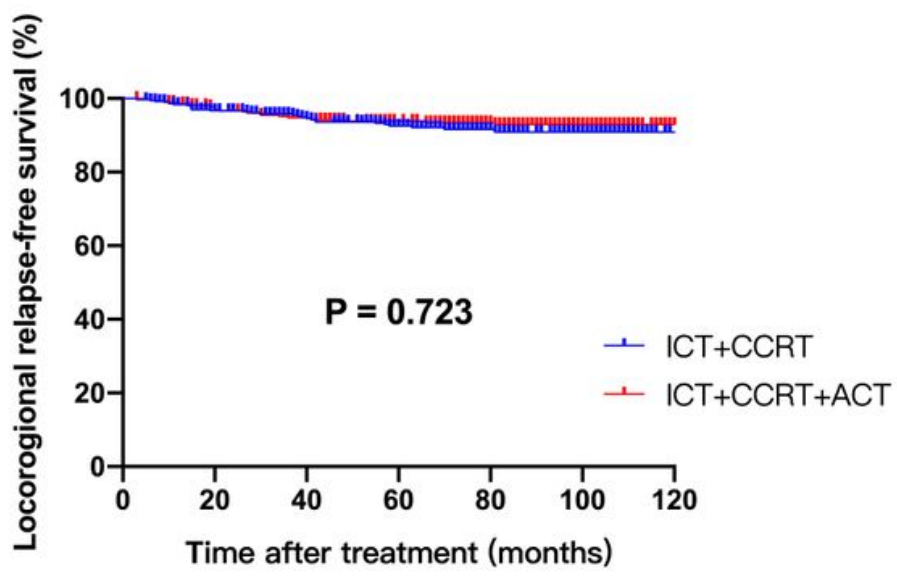

Fig.2 Kaplan-Meier Analysis of Overall survival, Disease-free survival, Distant Metastasis-free survival and Locoregional relapse-free survival.

Figure 2

See image above for figure legend. 
A

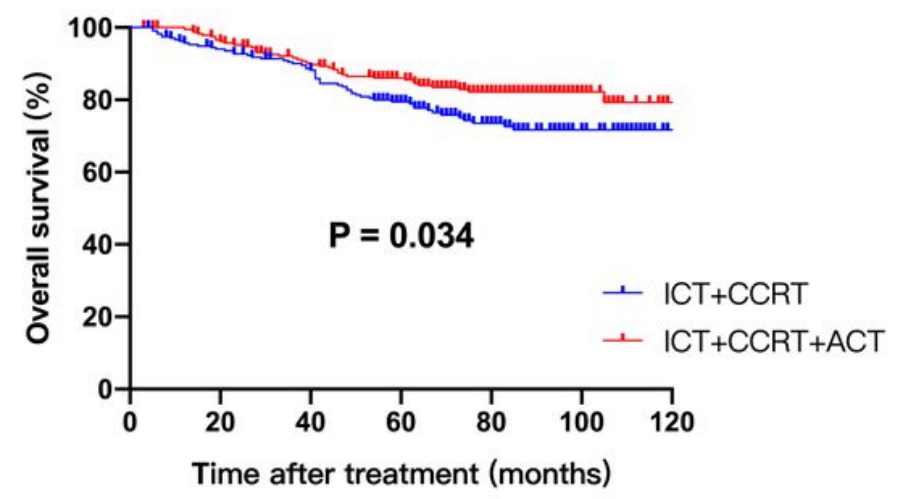

C

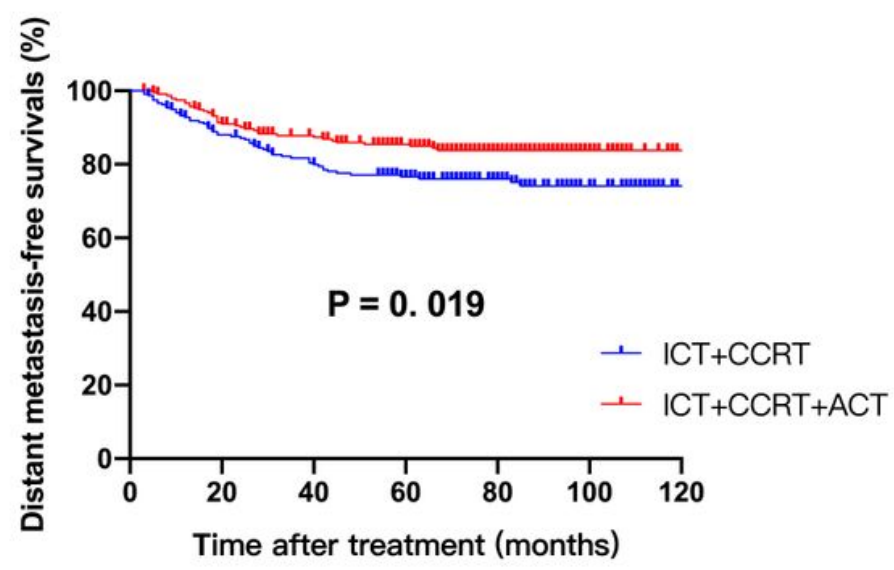

B

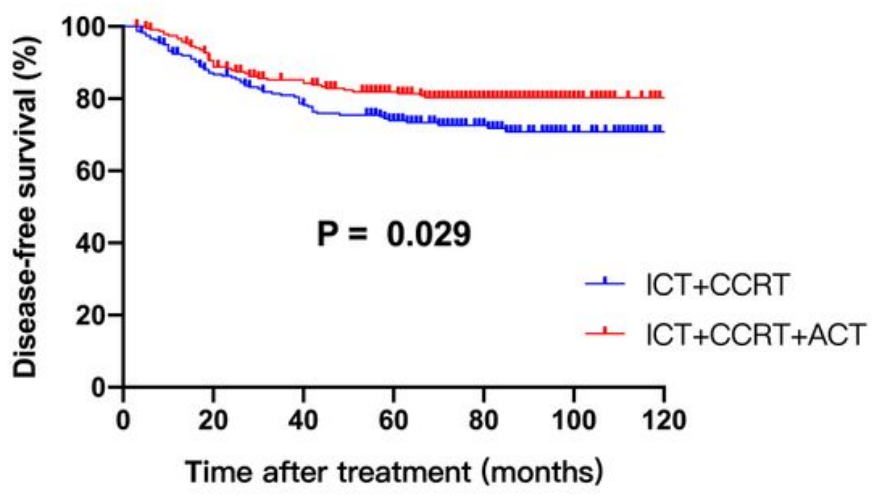

D

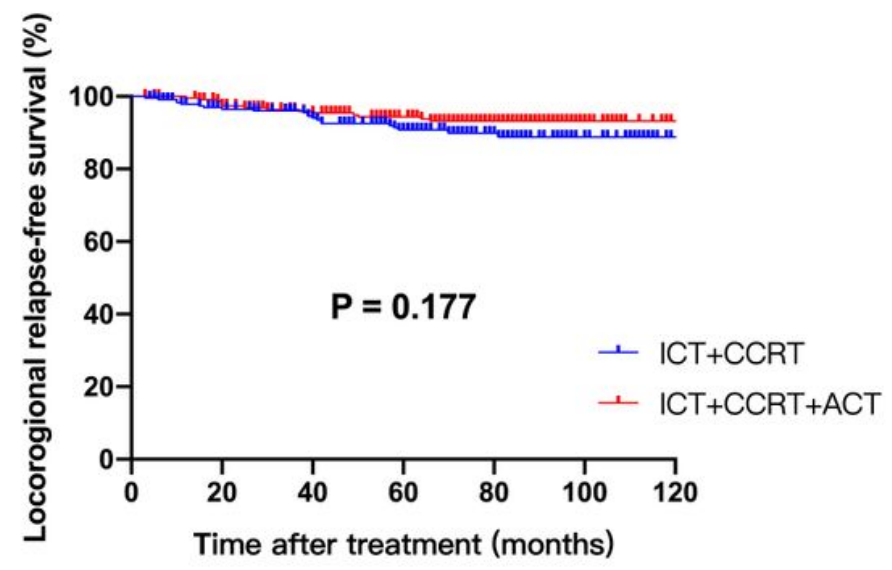

Fig.3 Kaplan-Meier Analysis of Overall survival, Disease-free survival, Distant Metastasis-free survival and Locoregional relapse-free survival.

Figure 3

See image above for figure legend. 